\title{
Revascularização direta do miocárdio sem circulação extracorpórea: estudo crítico dos resultados em 391 pacientes
}

\author{
Ênio BUFFOLO*, José Carlos S. ANDRADE*, José Ernesto SUCCI*, Luiz E. V. LEÃO*, João Nelson \\ Rodrigues BRANCO*, Clotário CUEVA*, Luciano F. AGUIAR*, Costabile GALLUCCI*, São Paulo, SP.
}

\begin{abstract}
RBCCV
BUFFOLO, E.; ANDRADE, J. C. S.; SUCCI, J. R.; LEÃO, L. E. V.; BRANCO, J. N. R.; CUEVA, C.; AGUIAR, L. F.; GALLUCCI, C. - Revascularização direta do miocárdio sem circulação extracorpórea: estudo crítico dos resultados em 391 pacientes. Rev. Bras. Cir. Cardiovasc., 1 (1): 32-39, 1986.

RESUMO: Os autores analisam os resultados obtidos em 391 pacientes consecutivos e não selecionados e submetidos a revascularização direta do miocárdio sem o circuito cardiopulmonar, operados de setembro de 1981 a fevereiro de 1985 . Neste mesmo periodo, foram realizadas um total de 1568 cirurgias de revascularizaçāo, sendo esta alternativa tática aplicável em $24,9 \%$ dos casos. Da casuística apresentada, 318 ( $81,3 \%$; eram do sexo masculino e $73(18,7 \%)$, do feminino e as idades de 35 a 76 anos, oscilando em torno da mediana 56. As condiçōes pré-operatórias indicativas da cirurgia foram as seguintes: insuficiência coronária crônica $286(73,1 \%)$ casos; pós trombólise coronária $42(10,7 \%)$; infarto agudo em evolução $18(4,6 \%)$; pós angioplastia mal sucedida $18(4,6 \%)$; reoperaçōes $14(3,6 \%)$; síndrome intermediária 12 $(3,1 \%)$ e revascularização em dissecção aórtica crônica 1 caso. Pontes únicas foram realizadas em 175 $(44,7 \%)$ pacientes, duplas em $192(49,1 \%)$ e triplas em $24(6,1 \%)$, com a média de 1,6 pontes/paciente. A artéria mamária foi usada para 109 casos, artéria mamária heteróloga bovina em 2, sendo, nos demais, utilizada a veia safena. A mortalidade hospitalar foi de 2,5\% (10/391), o que difere, significativamente, da mortalidade de $5 \%$ verificada para um grupo de 1177 pacientes operados no mesmo período, com a circulação extracorpórea. Comparamos, prospectivamente, as complicaçōes pós-operatórias observadas em um grupo de 378 pacientes operados sem a circulação extracorpórea, contra um grupo controle de 689 pacientes tratados da maneira convencional, no que diz respeito à incidência de arritmias, infarto per-operatório, complicaçōes pulmonares, neurológicas, infecciosas e hemorrágicas. Verificamos uma incidência menor, estatisticamente significante, para as complicaçōes pulmonares, neurológicas e de arritmias no grupo operado sem a circulação extracorpórea, não diferindo das demais complicaçōes entre os dois grupos. Concluem os autores que a revascularização do miocárdio sem utilização da extracorpórea se consolidou, ao longo destes 4 anos de experiência, çomo tática de eleição em lesões da coronária direita, descendente anterior, ou ambas, face às vantagens que apresenta.
\end{abstract}

DESCRITORES: Miocárdio, revascularização direta; circulaçāo extracorpórea.

Trabalho realizado na Disciplina de Cirurgia de Tórax da Escola Paulista de Medicina, São Paulo, SP. Brasil

Apresentado ao 13: Congresso Nacional de Cirurgia Cardiaca, São Paulo, SP, 4 e 5 de abril, 1986.

- Da Escola Paulista de Medicina.

Endereço para Separatas: Ênio Buffolo, Rua Borges Lagoa, 783. São Paulo, SP, Brasil.

*. D. Effler. Comunicação pessoal. Syracuse, N. Y., abril 1984. 


\section{INTRODUÇÃO}

Apesar das primeiras tentativas da realização de pontes de safena aorto-coronárias terem sido realizadas sem o auxílio do circuito cardiopulmonar, logo após esta reduzida experiência inicial, ficou evidente que a realização das anastomoses safeno-coronárias poderiam ser realizadas com maior facilidade e melhor qualidade com o coraçāo em assistolia sob circulação extracorpórea ${ }^{\star * 5.6}$.

Nos anos subseqüentes, observamos um extraordinário esforço de cardiologistas e cirurgiōes cardiovasculares, no sentido de baixar consideravelmente o risco operatório e a morbidade, assim como assegurar indices de permeabilidade imediata e tardia aceitáveis. Estes objetivos básicos foram atingidos plenamente, fazendo com que a revascularização cirúrgica do miocárdio se consolidasse como um procedimento real e efetivo para um grande contingente de coronariopatas.

Apesar de, na atualidade, o risco operatório ser baixo e aceitável, a morbidade não é pequena, especialmente para grupos especiais de pacientes, fazendo com que os grupos cirúrgicos procurem otimizar as técnicas do suporte cardiopulmonar e os cuidados pós-operatórios.

Sendo a cirurgia de revascularização do miocárdio um procedimento realizado na superfície do coração, sem a abertura das suas câmaras, seria intuitivo e logístico que se procurassem alternativas táticas de feitura de pontes aorto-coronárias com o coração batendo e com a manutenção da ventilação e circulação fisiológicas.

Entusiasmados com os resultados iniciais obtidos em um grupo de pacientes revascularizados sob circuito extracorpóreo parcial e com o coração batendo, decidimos eliminar, completamente, o auxílio cardiopulmonar para a realização de anastomoses safeno-coronárias com artérias acessiveis pela abordagem mediana longitudinal, quais sejam a descendente anterior, coronária direita, ou ambas.

Esta tática já havia sido realizada anteriormen$t^{7}{ }^{7}$, mas não teve, a nosso ver, maior divulgação e aplicação, pela necessidade, no conceito dos autores, de se realizar perfusão coronária proximal e distal à zona da interrupção do fluxo, devido ao temor e risco do infarto intraoperatório.

Com o advento mais recente de potentes drogas, que diminuíram o consumo de $\mathrm{O}_{2}$ do miocárdio, nos pareceu seguro interromper, temporariamente, a circulação coronária, após a administração de drogas bloqueadoras dos canais de $\mathrm{Ca}$, e não realizar nenhum artifício de perfusão coronária durante a anastomose. Os resultados iniciais bastante satisfatórios, assim como descrição da técnica operatória, foram publicados anteriormente, demonstrando vantagens em relação ao grupo de pacientes operados com circulação extracorpórea ${ }^{2,3,4}$.

O presente trabalho pretende, de maneira critica, analisar a experiência atual com um total de 391 pacientes e discutir as vantagens e desvantagens desta alternativa tática de revascularização do miocárdio, em relação ao procedimento convencional.

\section{CASUÍSTICA E MÉTODO}

De outubro de 1981 a fevereiro de $1986^{*}$, foram operados 391 pacientes, sendo 318 do sexo masculino e 73 do feminino, com idades variando de 35 a 76 anos, oscilando em torno da mediana 56 .

Foram selecionados, para a revascularização miocárdica sem extracorpórea, apenas pacientes com lesões obstrutivas no território da descendente anterior, ou ambas, sendo que esta seleção era feita pré-operatoriamente, com base na análise da cinecoronariografia e excluindo-se da programação pacientes com lesões no território da circunflexa, artérias com parede extremamente doente, ou demasiado finas e, ainda, artéria descendente anterior de trajeto intramiocárdico. No mesmo período, foram operados pelo nosso grupo 1568 pacientes, mostrando-se a revascularização sem extracorpórea aplicável em $24,9 \%$ dos casos.

Os critérios clínicos indicativos da revascularização miocárdica encontram-se na Tabela 1, chamando a atenção o percentual expressivo de pacientes operados na fase aguda de insuficiência coronária (pós estreptoquinase, infarto agudo em evolução, síndrome intermediária e, ainda, angioplastia mal sucedida).

TABELA I

Critérios clínicos da indicaçâo operatória

\begin{tabular}{lrc}
\hline \hline & N: & $\%$ \\
\hline Insuficiência coronária crônica & 286 & $(73,1 \%)$ \\
Pós-estreptoquinase & 42 & $(10,7 \%)$ \\
Infarto agudo em evoluçāo & 18 & $(4,6 \%)$ \\
Pós-angioplastia mal sucedida & 18 & $(4,6 \%)$ \\
Reoperaçōes & 14 & $(3,6 \%)$ \\
Síndrome intermediária & 12 & $(3,1 \%)$ \\
Aneurisma dissecante & 1 & $(0,3 \%)$ \\
& 391 & $(100 \%)$ \\
\hline
\end{tabular}

- Casuística dos Hospitais: Unicor, do Coração, São Paulo e Albert Einstein (São Paulo, SP).

-. Bioenxerto Biocor. 
Pontes únicas foram realizadas em 175 $(44,7 \%)$ pacientes, duplas em $192(49,1 \%)$ e triplas em $24(6,1 \%)$, com um total de 631 pontes, nos 391 pacientes operados, e média de 1,6 pontes/ paciente.

Para a realização dos desvios aorto-coronários, a veia safena foi utilizada 520 vezes, a artéria mamária 109 e, finalmente, a artéria mamária heteróloga bovina** em 2 situações.

Quanto ao tipo de artérias coronárias tratadas, a descendente anterior foi a mais comumente abordada, 361 vezes, vindo, a seguir, a coronária direita 190 , ramo diagonal 71 , descendente posterior 7 e, finalmente, ramo diagonalis 2 vezes.

\section{DESCRIÇÃO DA TÉCNICA}

Os pormenores da técnica cirúrgica utilizada já foram publicados anteriormente ${ }^{3,7}$. A toracotomia mediana longitudinal é realizada da maneira convencional, conseguindo-se ampla exposição da face anterior do coração, com generosa abertura do pericárdio em direção a ambos os seios cardiofrênicos e com tração mais acentuada do pericárdio através de seis ou oito pontos aplicados em seus bordos.
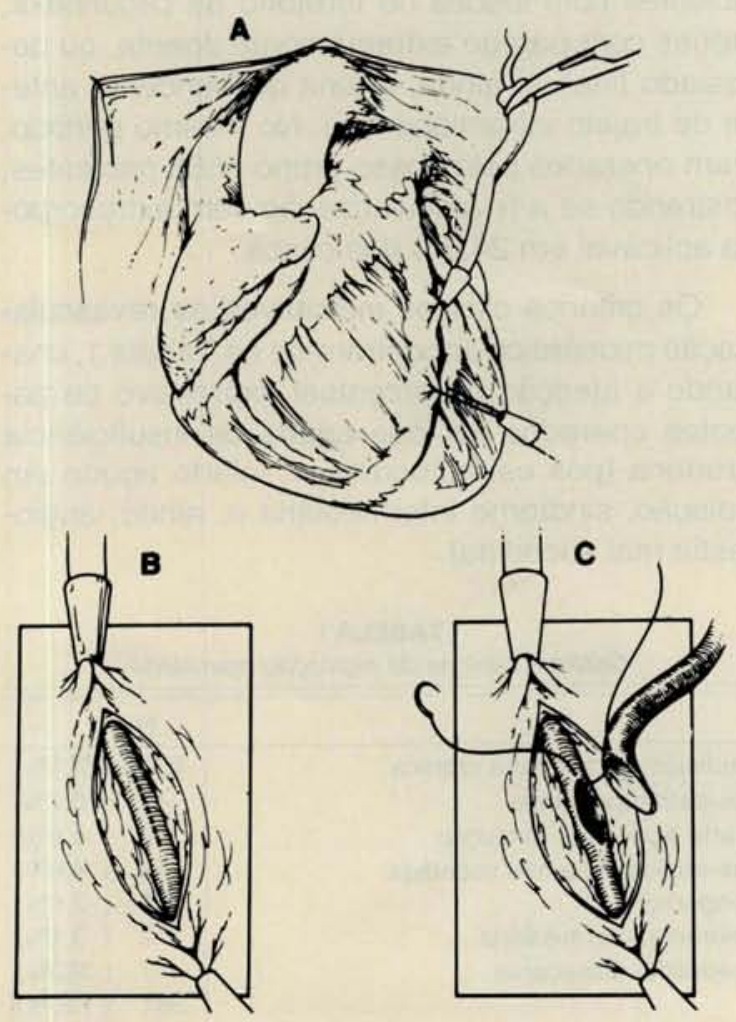

Após inspeção da rede arterial coronária subepicárdica, dissecamos os segmentos coronarianos no local a ser feita a anastomose (habitualmente, a descendente anterior, ao nivel do seu terço médio, ou a direita distal, pouco antes da descendente posterior).

A heparinizaçâo é, entâo, efetuada com $2 \mathrm{mg} /$ $\mathrm{kg}$ peso, sendo, a seguir, administrado verapamil intravenoso na dosagem de $5 \mathrm{mg}$ imediatamente antes da oclusão coronária, repetindo-se esta administração na mesma dose, se houver necessidade de outras anastomoses em outros ramos coronários.

Dissecada a coronária, aplicamos pontos prolene 5-0 proximal e distalmente, com a finalidade de, com o garroteamento através de delicados tubos de silicone, realizar a hemostasia (Figura 1).

Incisada a coronária na extensão aproximada de 8 a $10 \mathrm{~mm}$, realizamos a anastomose da safena, ou mamária, com fios de prolene 7-0 em sutura continua. Terminada a anastomose, verificamos a hemostasia, libetando-se o fluxo da mamária, ou com injeção de soro fisiológico aquecido fisiologicamente no segmento de safena.

Nos casos de pontes aorto-coronárias com a veia safena, a anastomose proximal é realizada sob o pinçamento lateral da aorta ascendente.
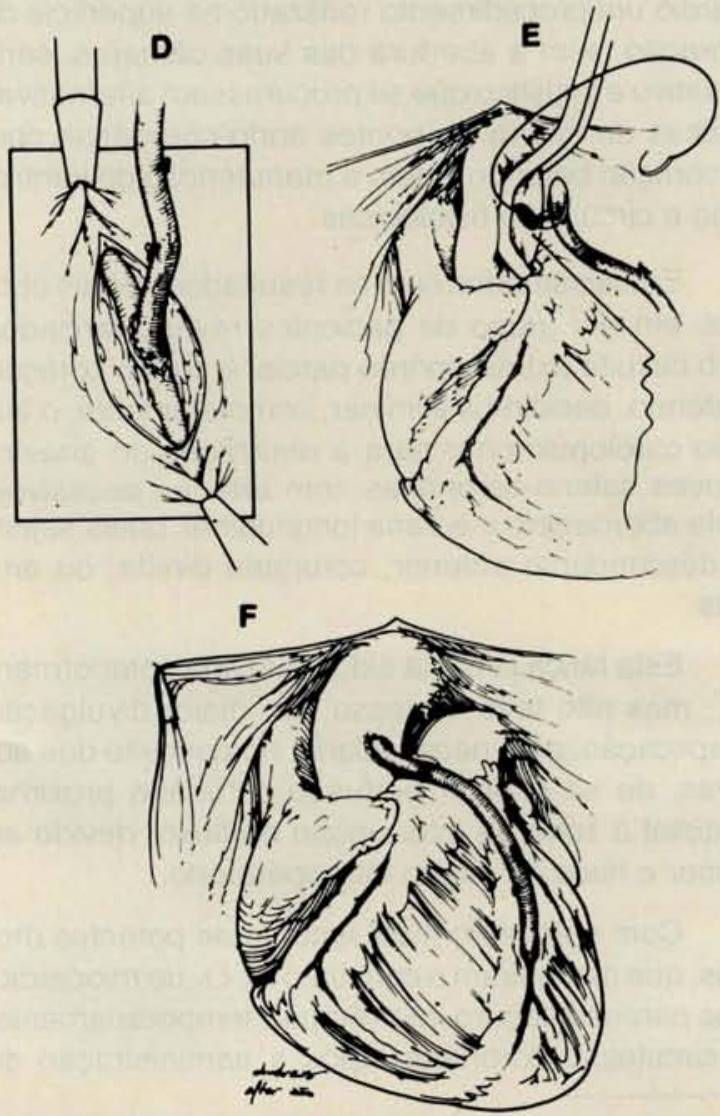

Fig. 1 - Desenho esquemático da sequeência dos tempos para realizaçâo de ponte de safena para a descendente anterior. 
Quando mais de uma ponte foi realizada, o garroteamento da artéria coronária subseqüente só era realizado após a liberação do fluxo da ponte precedente.

Terminadas as anastomoses e verificada a hemostasia, procedemos à neutralização da heparina com protamina na relação 1:1.3.

A drenagem e o fechamento da parede torácica foram realizados da maneira convencional, sem manobras especiais.

Todos os pacientes, após o ato cirúrgico, foram removidos à unidade de terapia intensiva, permanecendo por 48 horas em média, onde, entre os controles habituais, eram realizados eletrocardiogramas diários, controles radiológicos e curva de liberação enzimática a cada 4 horas, nas primeiras 24 horas.

Foi, ainda, desenvolvido um programa especial de computador (sistema "d Base"), para analisar todas as complicações pós-operatórias da fase hospitalar, sendo que este programa aplicável, prospectivamente, a um grupo de 378 casos operados sem circulação extracorpórea, cotejando-o com um grupo controle de 689 pacientes revascularizados no mesmo período. A incidência destas complicações, tabuladas sob a forma de 10 colunas com múltiplas combinações alfanuméricas, foram analisadas estatisticamente pelos testes $T$ de Student, quanto à sua significância, sendo que, em todos os testes, fixamos em 0,05 , ou $5 \%$, o nível para a rejeição da hipótese de nulidade, sendo assinalado com arterisco os valores significantes.

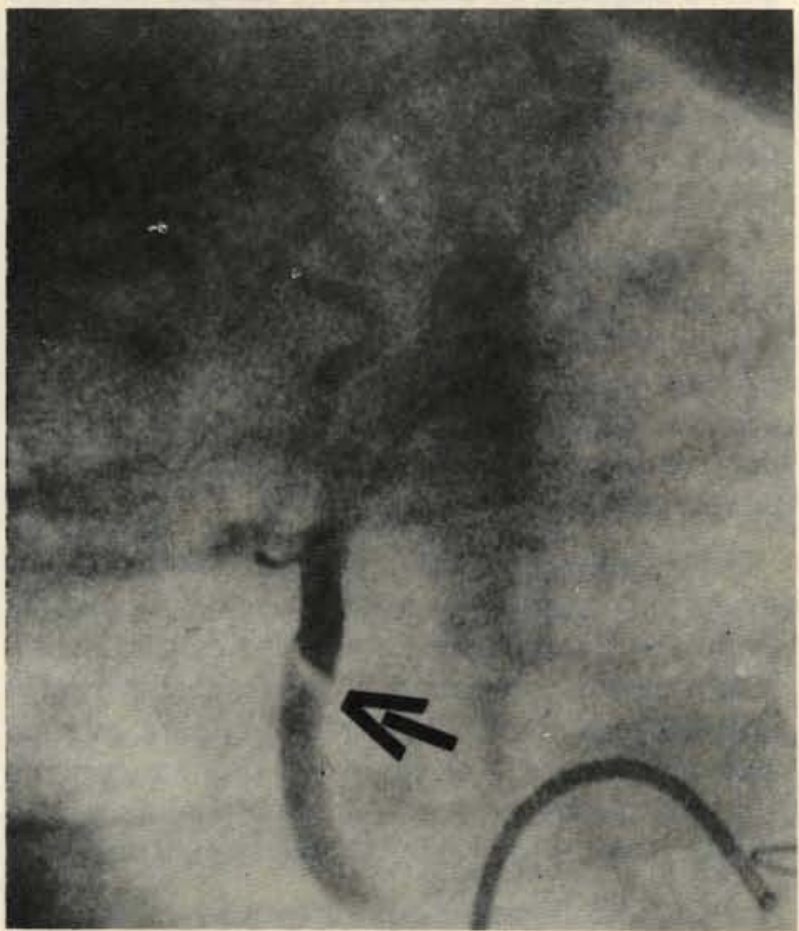

\section{RESULTADOS}

Nesta série de 391 pacientes, ocorreram 10 óbitos hospitalares $(2,5 \%)$, sendo as causas de óbito: septicemia, mediastinite, choque cardiogênico, infarto do miocárdio, bloqueio $\mathrm{A}-\mathrm{V}$ total, aneurisma dissecante, embolia pulmonar, trombose mesentérica, morte súbita de etiologia indefinida e, finalmente, fibrilação ventricular refratária. Esta mortalidade $(2,5 \%)$ foi estatisticamente inferior à observada no grupo controle de 1177 pacientes revascularizados no mesmo período $(5 \%)$.

Entre todas as complicações pós-operatórias analisadas, destacamos as de maior relevância, quais sejam: arritmias, pulmonares, infarto do miocárdio, neurológicas, hemorrágicas e infecciosas, comparando a incidência percentual em dois grupos com e sem circulação extracorpórea, estando assinaladas em asteriscos as que têm diferença estatisticamente significante (Tabela 2).

TABELA 2

Complicaçōes pós-operatórias observadas na revascularização do miocárdio

\begin{tabular}{lcc}
\hline \hline & $\begin{array}{c}\text { Grupo I } \\
\text { com CEC } \\
698 \text { casos }\end{array}$ & $\begin{array}{c}\text { Grupo II } \\
\text { sem CEC } \\
378 \text { casos }\end{array}$ \\
\hline Arritmias* $^{*}$ & $12,6 \%$ & $5,5 \%$ \\
Pulmonares* $^{*}$ & $9,7 \%$ & $3,2 \%$ \\
Infarto do Miocárdio & $4,4 \%$ & $4,8 \%$ \\
Neurológicas* & $3,8 \%$ & $1,1 \%$ \\
Hemorrágicas & $3,5 \%$ & $4,8 \%$ \\
Infecciosas & $3,3 \%$ & $3,7 \%$ \\
\hline
\end{tabular}

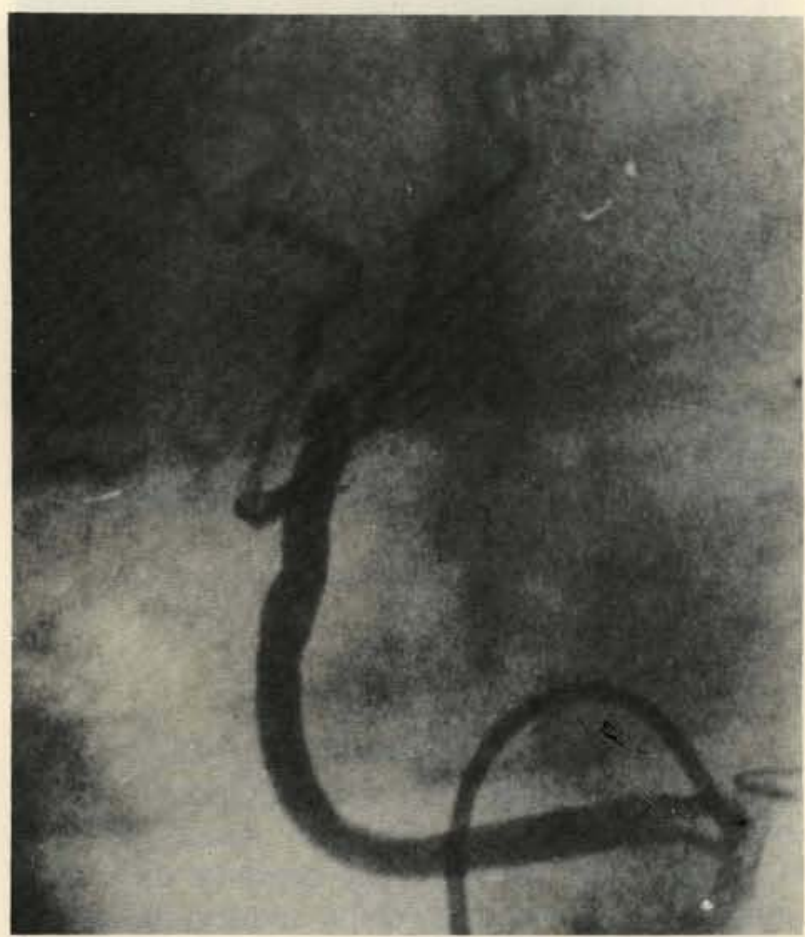

Fig. 2 - Estudo controle do paciente com ponte de safena para a descendente anterior. 
Observe-se que as complicações pulmonares, neurológicas e arritmias mais graves foram mais freqüentes no grupo operado com circulação extracorpórea, sendo as demais complicações não diferentes.

O infarto per-operatório ocorreu com incidências comparáveis $(4,4 \%$ e $4,8 \%)$ nos dois grupos.

Dos 381 pacientes que receberam alta hospitalar e foram acompanhados por um período médio de 25 meses, ocorreram 2 óbitos tardios $(0,5 \%)$ (1 mês de pós-operatório, broncopneumonia e 3 meses, morte súbita) e 5 reoperações $(1,3 \%)$ por obstrução de ponte, ou, ainda, estenose ao nível da anastomose.

Cineangiocoronariografias controles foram realizadas em 51 pacientes $(13,0 \%)$, sendo, em 23 , por sintomas residuais em períodos de tempo variáveis de 3 a 38 meses e, em 28, para investigação científica antes da alta hospitalar. Nestes grupos, foram estudadas 77 pontes, ou seja $12,2 \%$ ( $77 / 631$ ), encontrando-se 66 pérvias $(81,8 \%)$ (Figuras 2 e 3) e 14 ocluídas, ou com estenose ao nível da sutura $(18,2 \%)$.

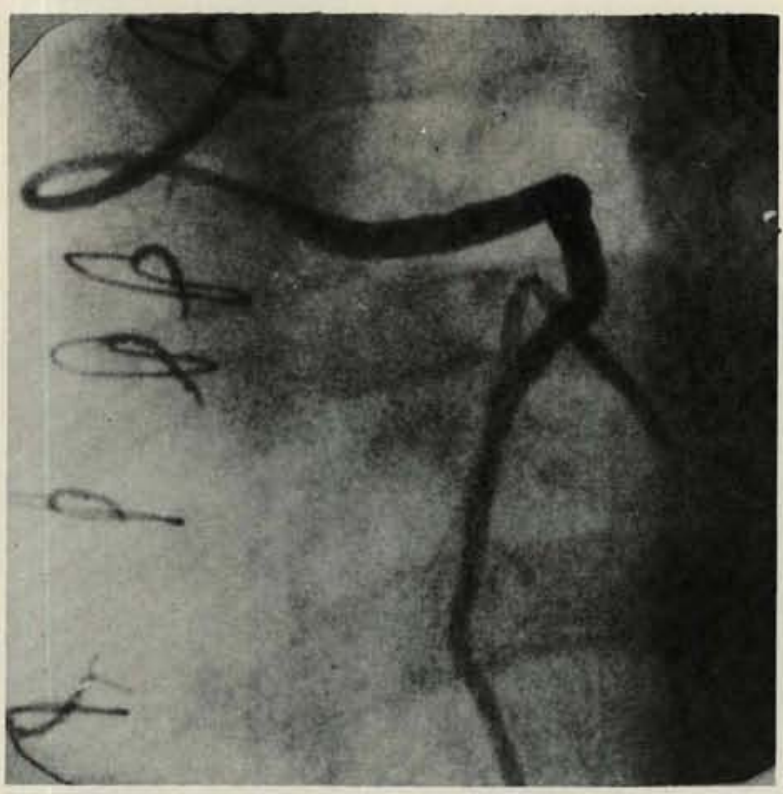

Fig. 3 - Ponte de passagem diagonal - descendente anterior.

Dos 14 pacientes portadores de anastomoses mamário-descendente anterior, em todos a ponte estava pérvia (100\%) (Figura 4), sendo que, em 1 , havia estenose na porção proximal da artéria mamária, possivelmente como seqüela da aplicação do bull-dog hemostático durante o ato cirúrgico.

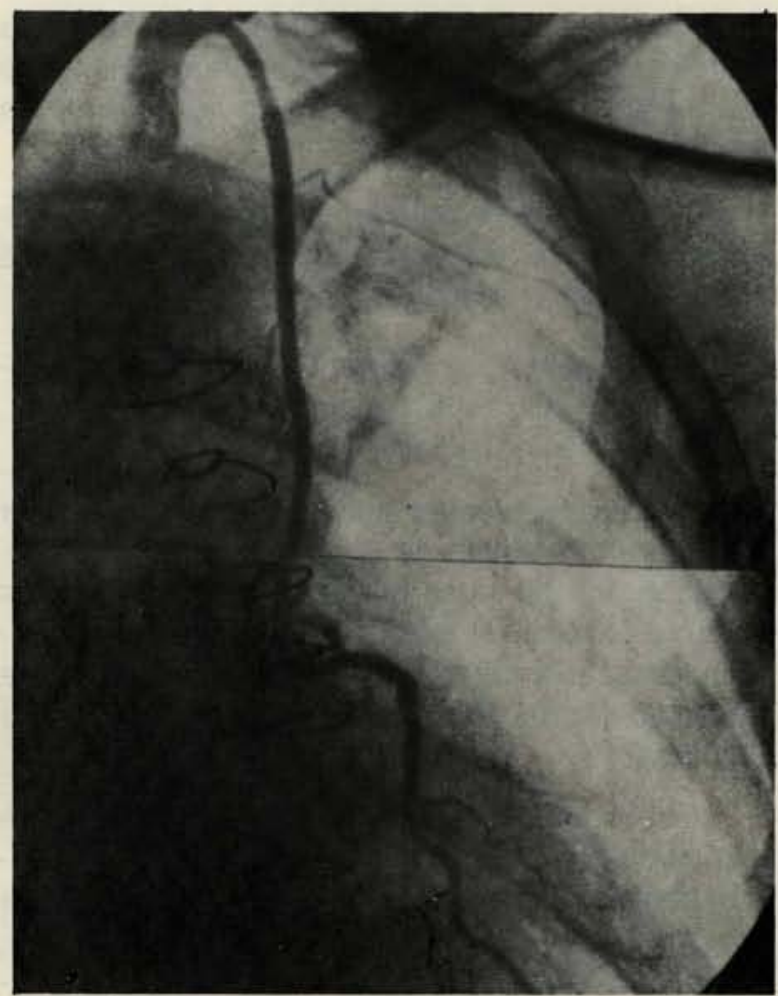

Fig. 4-Anastomose mamária-descendente anterior pérvia

\section{DISCUSSÃO}

As vantagens da realização de pontes aortocoronárias sem a utilização do circuito cardiopulmonar são intuitivas. Entre estas vantagens, citamos a maior simplicidade do procedimento cirúrgico, a dispensa da utilização de sangue homólogo e a indiscutível vantagem econômica pela não utilização da máquina coração-pulmão e os oxigenadores descartáveis, fazendo com que a revascularização do miocárdio tenha a equivalência de uma toracotomia.

Já tínhamos observado, em trabalhos anteriores, que: o tempo médio de internação hospitalar era menor (7 dias), comparado com grupo de pacientes operados da maneira convencional ( 11 dias), a incidência de complicaçōes pós-operatórias era menor e havia sugestão de menor risco de mortalidade hospitalar.

Neste trabalho, com maior casuística e seguimento pós-operatório, os dados avaliados prospectivamente e sob a crítica da análise estatística permitem concluir que a mortalidade hospitalar é significantemente menor, 2,5\% (10/391) para o grupo sem extracorpórea do que para o grupo controle de 1177 pacientes operados da maneira convencional, onde encontramos $5 \%$ de mortalidade.

Cumpre ressaltar que os grupos incluem pacientes consecutivos e não selecionados, sendo 
que, no grupo de pacientes operados sem extracorpórea, alguns pacientes muito graves tiveram a indicaçāo cirúrgica formalizada pelo fato de poderem ser operados sem o circuito cardiopulmonar, incluindo insuficiência renal grave na vigência de hemodiálise, disfunção pulmonar acentuada, infarto agudo em evolução e aneurisma dissecante, entre outras.

Por outro lado, os grupos diferiam quanto ao número de vasos lesados, sendo, no grupo sem extracorpórea, predominantes em 1 ou 2 vasos; no outro grupo, havia quase que exclusivamente lesōes de mais de 2 vasos. Os grupos não diferiam basicamente quanto à forma clínica de apresentação da insuficiência coronária, ou, ainda, no grau de comprometimento ventricular, sendo, conseqüentemente, a nosso ver, a menor mortalidade hospitalar observada, resultado direto da tática de revascularização utilizada.

Após a experiência inicial, pareceu-nos de importância demonstrar, objetivamente, a diferença da incidência de complicações pós-operatórias entre os dois grupos, o que já havia chamado a atenção na observação clínica dos pacientes.

Fizemos, então, um estudo prospectivo, no mesmo período de tempo, com a mesma equipe cirúrgica e em condiçōes hospitalares semelhantes, analisando, $\in m$ sistema "d Base", todas as complicações pós-operatórias observadas. De todas as complicaçōes tabuladas, resumimos, na Tabela 2 , as mais relevantes, incluindo-as em 6 grandes grupos: arritmias, pulmonares, infarto do miocárdio, neurológicas, hemorrágicas e infecciosas.

As arritmias assinaladas incluem apenas distúrbios de rítmo mais sérios, tais como: fibrilaçāo atrial, taquicardias paroxísticas, rítmos funcionais, fibrilação ventricular, entre outras, não se considerando extrassistolias ocasionais, na casuística.

O infarto per-operatório teve, como critério diagnóstico, a presença de ondas $Q$ de necrose adicionais e/ou curva enzimática alterada, com elevação dos valores de CKMB acima de $20 \mathrm{U}$.

As complicaçōes hemorrágicas consideradas na estatística foram aquelas que levaram à reoperação para revisão da hemostasia.

Verifica-se, pela análise dos resultados, que a incidência de arritmias, complicaçōes pulmonares e neurológicas foram estatísticamente mais prevalentes no grupo de 689 pacientes operados com circulação extracorpórea, não se observando diferenças entre os grupos para a incidência de infarto per-operatório, complicações hemorrágicas, ou infecciosas.
A análise desta experiência permite, pois, a conclusão de que a revascularização miocárdica realizada sem o circuito cardiopulmonar é intervenção cirúrgica de menor risco e morbidade.

Uma crítica relevante que se pode fazer a este procedimento diz respeito à maior dificuldade técnica na feitura das anastomoses mamário, ou safenocoronárias, com o coração em movimento, o que poderia comprometer a qualidade da anastomose e o resultado da revascularização.

Concordamos que existe, realmente, maior dificuldade na realização das suturas, sendo, por este motivo, não recomendável para cirurgiōes em início de formaçāo. Todavia, com manobras especiais de luxação do coração, do saco pericárdico e hemostasia cuidadosa, é perfeitamente possivel a realização das anastomoses, sem grandes dificuldades para cirurgiōes de reconhecida experiência com a revascularização direta.

Os nossos resultados em reestudos controles demonstram permeabilidade de $81,8 \%$ para as pontes de safena e $100 \%$ de permeabilidade para as 14 anastomoses mamário-descendente anterior.

Estes números são aceitáveis, se considerarmos que 23, dos 51 pacientes reestudados, tiveram suas pontes avaliadas por sintomas residuais, o que onera os indices de permeabilidade. É de se esperar que índices maiores seriam obtidos se o estudo incluisse maior percentual de assintomáticos avaliados em estudos de rotina.

Acreditamos, pois, que as pontes com a artéria mamária, ou safena, podem ser realizadas com a mesma qualidade técnica da obtida com o coraçāo em assistolia. Outros autores, todavia, observaram, também, em casuísticas expressivas, meIhores resultados no tratamento da coronária direita do que para a descendente anterior, considerando esta alternativa tática ideal para as angioplastias mal sucedidas.

Face à experiência acumulada ao longo destes 5 anos, acreditamos ser a revascularização miocárdica sem circulação extracorpórea alternativa tática de eleição para um determinado grupo de pacientes portadores de lesões coronárias, podendo oferecer menor risco operatório e menor incidência de complicações pós-operatórias.

\section{CONCLUSÕES}

1) A revascularização do miocárdio sem circulação extracorpórea é aplicável, em nossas condições de trabalho, em cerca de $1 / 4$ dos pacientes $(24,9 \%)$. 
2) A mortalidade hospitalar foi mais baixa $(2,5 \%)$, quando comparada a um grupo controle revascularizado no mesmo período $(5 \%)$.

3) Dentre as complicações pós-operatórias analisadas prospectivamente em dois grupos: Gru-
2 BUfFOLO, E.; ANDRADE, J. C.; SUCCI, J.; LEĀO, L. E.; CUEVA, C.; BRANCO, J. N. R.; CARVALHO, A. C.; GALLUCCI, C. - Direct myocardial revascularization without extracorporeal circulation: technique and initial results. Texas Heart Inst. J., 12: 33-41, 1985.

BUFFOLO, E.: ANDRADE J. C. S.; LEÃO, L. E. V.; BRANCO, J. N. R.; CUEVA, C.: AGUiAR,

L. F.; GALLUCCI, C. - Direct myocardial revascularization without extra-corporeal circulation: results in 391 patients. Rev. Bras. Cir. Cardiovasc., 1 (1): 32-39, 1986.

ABSTRACT: The authors analyse the results of 391 consecutive non-selected patients who underwent direct myocardial revascularization (MR) without cardiopulmonary bypass (CPB) from September 1981 to February 1985 . In the same period we performed a total of 1568 revascularization procedures; thus $24.9 \%$ were MR without CPB. Out of the 391 patients, $318(81.3 \%)$ were male and the ages varied from 35 to 76 , with a median of $56 \mathrm{y} / \mathrm{o}$. The surgical procedure was performed due to: chronic coronary insufficiency $286(73.1 \%)$, after coronary thrombolysis $42(10.7 \%)$, evolving acute myocardial infarction $18(4.6 \%)$, ill-succeeded angioplasty $18(4.6 \%)$, redos $14(3.6 \%)$, intermediate syndrome $12(3.1 \%)$ and chronic aortic dissection 1. Single bypass was performed in $175(44.7 \%)$ patients, double in $192(49.1 \%)$ and triple in $24(6.1 \%)$ with an average of 1.6 grafts/patient. The mammary artery was utilized 109 times, bovine heterologous mammary 2 and all remaining cases received saphenous vein grafts. The hospital mortality was $2.5 \%(10 / 391)$ which is significantly different from our $5 \%$ hospital mortality in 1177 patients operated on the same period with CPB. We prospectively compared the postoperative complications in 378 patients without CPB against 689 patients with CPB concerning arrhythmias, perioperative myocardial infarction, neurologic, infectious, pulmonary and hemorrhagic complications.

DESCRIPTORS:myocardial revascularization, direct; extracorporeal circulation.

po I, 689 pacientes com CEC e Grupo II, 378 sem CEC, as complicações neurológicas, pulmonares e arritmias foram significantemente maiores no Grupo I.

4) A revascularização do miocárdio sem circulação extracorpórea se constitui numa alternativa eficiente e segura de tratamento da insuficiência coronária.

\section{REFERÊNCIAS BIBLIOGRÁFICAS}

1 ARCHER, R.; OTT, D. A.; PARRAVICINI, R.; COOLEY, D. A.; REUL, G. J.; FRAZIER, O. H.; DUNCAN, J. M.; LIVESAY, J. J.; WALKER, W. E. - Coronary artery revascularization without cardiopulmonary by-pass. Texas Heart Inst. J., 11: 52-57, 1984.
3 BUFFOLO, E.; ANDRADE, J. C. S.; SUCCI, J. E.; LEĀO, L. E. V.; CUEVA, C.; BRANCO, J. N. R.; CARVALHO, A. C. C.; GALLUCCI, C. - Revascularização direta do miocárdio sem circulação extracorpórea: descrição da técnica e resultados iniciais. Arq. Bras. Cardiol. 41: 309-316, 1983.

4 BUFFOLO, E.; ANDRADE, J. C.; SUCCI, J.; LEĀO, L. E.; GALLUCCI, C. - Direct myocardial revascularization without cardiopulmonary bypass. Thorac. Cardiovasc. Surg., 33: 26-29, 1985.

5 FAVALORO, R. G. - Saphenous vein autograft replacement of severe segmental coronary artery occlusion: operative technique. Ann. Thorac. Surg., 5: 334-339, 1968.

6 JOHNSON, W. D.; FLEMMA, R. J.; LEPLEY Jr., D.; ELLISON, E. H. - Extended treatment of severe coronary artery disease: a total surgical approach. Ann. Surg., 170: $460-470,1969$.

7 TRAPP, W.G.; \& BISARYA, R. - Placement of coronary artery bypass graft without pump oxigenator. $A n n$. Thorac. Surg., 19: 1-9, 1975. 


\section{Discussão}

DR. REGIS JUCÁ

Fortaleza, CE

Gostaríamos de cumprimentar o Dr. Buffolo e seus associados por mais esta criativa contribuição científica.

Entretanto, não temos usado a técnica empregada pelos autores, porque, com o uso convencional da circulação extracorpórea, temos obtido bons resultados na cirurgia de revascularização do miocárdio. Nos primeiros 100 casos, a partir de outubro de 1972, quando iniciamos este tipo de cirurgia no Hospital de Messejana, a nossa mortalidade foi de $4 \%$, sem seleção de pacientes. Entre 1983 e 1985, em um de nossos Serviços (Casa de Saúde São Raimundo), operamos 193 pacientes, com 3 óbitos (mortalidade de 1,5\%). No ano passado, operamos 113 pacientes, com 2 óbitos (mortalidade de $1,76 \%$ ), sendo que um dos óbitos foi após uma reoperação com extensa endarterectomia da descendente anterior e, no outro, um pulmão super-inflado, devido a enfisema pulmonar, prejudicou a anastomose mamária - DA. Recentemente, influenciados pelos trabalhos de Buffolo e col. e de Akins, do Massachusetts General Hospital, tivemos que reoperar um paciente com angina instável, cuja pneumopatia grave impedia o emprego da circulação extracorpórea. F.R.R., com 43 anos, em 1973, recebeu dupla ponte de safena para uma coronária direita $(C D)$ dominante e para uma descendente anterior (DA) de fino calibre. Evoluiu bem, por 12 anos, do ponto de vista cardiaco. Em setembro de 1985 , devido a angina instável recurrente, foi submetido a cinecoronariografia, que revelou oclusão total da ponte para DA e estenose proximal da ponte para $C D$. $O$ quadro pulmonar com bronquiectasias, febre e expectoração contra-indicava o uso de circulação extracorpórea. Em outubro de 1985 , foi realizada nova ponte de safena da aorta para o enxerto da coronária direita, distal à obstrução, no terço médio. Durante o ato cirúrgico, o paciente recebeu altas doses de dopamina e xilocaina. O curso pós-operatório foi tormentoso, devido ao quadro pulmonar; recebeu alta no 10 : dia pós-operatório, ainda em regime de antibióticos. Um mês após a alta, não apresentava angina do peito, mesmo depois de longas caminhadas. A apresentação do caso justifica-se por:

1) possibilidade de emprego da técnica em casos similares, conforme sugerido por Buffolo e col.

2) estudo da função ventricular, 12 anos após 64 minutos de parada cardíaca em normotermia e sem soluções cardioplégicas (fração de ejeção $55 \%)$.
PROF. ADIB D. JATENE

São Paulo, SP

Gostaria de fazer um comentário adicional. $\mathrm{O}$ número de artérias lesadas é maior, para o grupo de pacientes operados com circulação extracorpórea. Isto sugere que os grupos são diferentes, sob o ponto de vista de doença coronária. Perguntaria se esta diferença do grau de comprometimento arterial não dificulta a comparação da mortalidade e das complicações pós-operatórias, nos dois grupos.

DR. BUFFOLO (Encerrando)

Agradecemos, inicialmente, o comentário do Dr. Jucá e aproveitamos a oportunidade para cumprimentá-lo pelos excelentes resultados obtidos, conseguindo operar com mortalidade hospitalar bastante baixa. Infelizmente, a nossa mortalidade nāo é esta; mesmo nos anos recentes, após extensa experiência com a revascularização miocárdica, atingimos uma estabilidade de cifras de mortalidade que, para pacientes consecutivos e não selecionados, oscila entre 4 e $5 \%$. Desta forma, a revascularização miocárdica sem a circulação extracorpórea, representou, para nosso ambiente de trabaIho, um progresso indiscutivel, no que diz respeito à diminuição do risco operatório. Para os grupos que conseguem realizar revascularização com risco cirúrgico inferior a $2 \%$, esta alternativa tática realmente não é atraente. Respondendo ao questionamento, aliás muito pertinente, do Dr. Adib, sobre a validade da comparação entre os dois grupos, acreditamos que eles, na realidade, o sejam, uma vez que não diferem basicamente quanto ao grupo etário e à função ventricular. Saliento, no entanto, que, se alguma diferença existe, quanto à situação clínica dos pacientes, ela é desfavorável em relação ao grupo operado sem extracorpórea. Estão incluídos, na casuística apresentada, pacientes que só tiveram a indicação cirúrgica confirmada pela possibilidade de serem operados sem extracorpórea, incluindo casos de doença pulmonar obstrutiva crônica de grau avançado, idades acima de 70 anos, com mau estado geral, pacientes extremamente obesos com peso acima de 100 quilos, ou, ainda, poucos casos de pacientes com insuficiência renal grave em programa de hemodiálise. Curiosamente, ainda enfatizamos que cerca de $22 \%$ dos pacientes desta série foram operados na fase aguda da insuficiência coronária, com revascularização pós trombótica, infarto em evolução, após angioplastia mal sucedida, ou, ainda, em síndrome intermediária, demonstrando que a casuistica não foi selecionada. 